\title{
Experimental Measurement and Numerical Modeling of the Effective Thermal Conductivity of TRISO Fuel Compacts
}

\author{
Charles Folsom ${ }^{\mathrm{a}}$, Changhu Xing ${ }^{\mathrm{a}}$, Colby Jensen ${ }^{\mathrm{a}}$, Heng Ban ${ }^{\mathrm{a},}$, and Douglas W. Marshall ${ }^{\mathrm{b}}$ \\ ${ }^{a}$ Mechanical \& Aerospace Engineering Department, Utah State University, Logan, UT 84322-4130 \\ ${ }^{\mathrm{b}}$ Idaho National Laboratory, Idaho Falls, ID 83415 \\ *+1.435.797.2098 heng.ban@ usu.edu, 4130 Old Main Hill, Logan, UT 84322-4130
}

\begin{abstract}
Accurate modeling capability of thermal conductivity of tristructural-isotropic (TRISO) fuel compacts is important to fuel performance modeling and safety of Generation IV reactors. To date, the effective thermal conductivity (ETC) of tristructural-isotropic (TRISO) fuel compacts has not been measured directly. The composite fuel is a complicated structure comprised of layered particles in a graphite matrix. In this work, finite element modeling is used to validate an analytic ETC model for application to the composite fuel material for particle-volume fractions up to $40 \%$. The effect of each individual layer of a TRISO particle is analyzed showing that the overall ETC of the compact is most sensitive to the outer layer constituent. In conjunction with the modeling results, the thermal conductivity of matrix-graphite compacts and the ETC of surrogate TRISO fuel compacts have been successfully measured using a previously developed measurement system. The ETC of the surrogate fuel compacts varies between $50-30 \mathrm{~W} \cdot \mathrm{m}^{-1} \cdot \mathrm{K}^{-1}$ over a temperature range of $50-600^{\circ} \mathrm{C}$. As a result of the numerical modeling and experimental measurements of the fuel compacts, a new model and approach for analyzing the effect of compact constituent materials on ETC is proposed that can estimate the fuel compact ETC with approximately $15-20 \%$ more accuracy than the old method. Using the ETC model with measured thermal conductivity of the graphite matrix-only material indicate that, in the composite form, the matrix material has a much greater thermal conductivity, which is attributed to the high anisotropy of graphite thermal conductivity. Therefore, simpler measurements of individual TRISO compact constituents combined with an analytic ETC model, will not provide accurate predictions of overall ETC of the compacts emphasizing the need for measurements of composite, surrogate compacts.
\end{abstract}

Key words: thermal conductivity, guarded-comparative heat flow technique, TRISO, numerical, experimental 


\section{Introduction}

Tristructural-isotropic (TRISO)-coated particle fuel will be used in Generation IV Very High Temperature Reactors (VHTR). TRISO fuel consists of TRISO-coated fuel particles $\approx 1 \mathrm{~mm}$ in diameter embedded in a graphite matrix. The "compact" form of the fuel is cylindrically shaped with a diameter of approximately $12.7 \mathrm{~mm}(0.5 \mathrm{in})$ and $25 \mathrm{~mm}$ (1 in) in length [1]. The TRISO particles consist of a fuel kernel that is coated with several layers: a porous pyrolytic carbon layer (buffer layer), a dense pyrolytic carbon (inner) layer, silicon carbide (SiC) layer, and another dense pyrolytic carbon (outer) layer [2]. An illustration of a TRISO fuel compact showing a cross-section view and a drawing of a TRISO particle is shown in Fig. 1. Although TRISO fuel has been used for many years, developmental efforts are ongoing in the United States, which require an understanding of the relationship between fuel fabrication and overall performance.

Due to the composite nature of the TRISO particles (as well as the graphite matrix itself), the overall ETC is needed. To date, the ETC of the composite TRISO fuel compacts has not been directly measured. Currently, fuel modeling for the VHTR utilizes a code called PARFUME (PARticle FUel ModEl) [3-4]. The ETC data used by the code are taken from a formula used by the German TRISO program from the late 1980's [5]. The formula uses measured thermal conductivity of matrix-only material and accounts for the effect of particles with a correction factor. The correction factor, $F_{p}$, is given as,

$$
F_{p}=\frac{1-\varphi}{1+\phi / 2}
$$

where $\square$ is the particle-packing fraction by volume. This correction factor represents a reduced form of Maxwell's ETC model [6] where the secondary phase has zero thermal conductivity. The assumptions of Maxwell's model require low particle-volume fractions $(<10 \%)$. The particle-volume fraction used by the German TRISO program were much smaller $(\sim 10 \%)$ than utilized by the current U.S. program $(\sim 30-40 \%)$ making the formalism of Eq. (1) less appropriate.

The objective of this work is to develop the most appropriate method of modeling the ETC of TRISO fuel compacts. In the ideal case, an analytic model could be used to predict overall ETC based on the graphite-matrix conductivity, measured using a standard such as laser flash, and the contribution of the particles. Recently, Stainsby et al. [7] investigated the ETC of TRISO fuel with a particle-volume fraction of $9.3 \%$ using finite-element analysis (FEA) for three different models: simple cubic (SC), body-centered cubic (BCC), face-centered cubic (FCC) unit cells. In this work, the study is extended to particle-volume fractions of up to $40 \%$. Additionally, a procedure is developed to generate randomly distributed particles as a model, which is more representative of TRISO fuel compacts. The FEA results are used to validate an analytic two-phase (graphite-matrix and particle) ETC model for TRISO fuel compacts. The effect of the particle constituents is included in the analysis. 
As a comparison to the numerical and analytic results, several surrogate TRISO compacts and matrix-only compacts are measured using a previously developed measurement system. Standard measurement systems such as laser flash cannot measure the ETC of the composite due to characteristic length scales of the composite fuel. The results from the experimental and numerical results are compared with the legacy German data and appropriate analytical effective conductivity models. The results show that prepared matrix-only samples are not good representatives of the matrix when prepared in composite form with particles. Thus, a new approach for analyzing the ETC of TRISO fuel compacts is proposed.

\section{ETC Modeling}

Many approaches to modeling ETC have been made in literature including analytic models [3, 8-9] and FEA models [10-12]. In most cases the analytical models are preferred over the numerical models due to their physical basis, low calculation cost, and reasonable accuracy even when microstructure is uncertain [9]. In this study, a combination of approaches is taken to better characterize the complex structure of the TRISO fuel compact.

\subsection{Binary Phase ETC Models}

Many ETC models have been developed in literature for predicting the ETC of heterogeneous materials (for a review see ref. [13]). Even with the great number of models available, the uncertainty in predicting the ETC of heterogeneous materials can still be quite high [12]. Many of the models are for specific materials and/or compositions and some require empirically derived parameters, therefore only a few of the models may apply to the fuel compacts.

The parameters affecting the ETC in heterogeneous materials are well defined in [14]. In the case of TRISO fuel compacts where the graphite matrix is considered continuous and the particles are uniform in size, the ETC, $k_{e}$, is a function of: the thermal conductivity of the graphite matrix, $k_{m}(T)$, the thermal conductivity of the particles (which are themselves a composite thus ETC is used, $k_{p}(T)$, the particlevolume fraction, $\square$, and in some cases the particle arrangement, $a$.

$$
k_{e}=k_{e}\left(k_{m}(T), k_{p}(T), \phi, a\right)
$$

Two useful parameters used in effective conductivity models are the ratio of the thermal conductivity of the dispersed phase (particles) to the continuous matrix,

$$
\kappa=\frac{k_{p}}{k_{m}},
$$

and by analogy with potential theory, the reduced polarizability,

$$
\beta=\frac{\kappa-1}{\kappa+2} \text {. }
$$


In this work, four models were chosen to apply to the TRISO fuel composite based on the model assumptions. These include: Maxwell's equation [6], the Reduced Maxwell's equation (Eq. (1)), Effective Medium Theory [15], and the Chiew and Glandt model [16]. A brief introduction to each of these models follows. Maxwell's model is one of the oldest, most well-known of ETC models and the basis of many later ETC models [9]. Using potential theory, the model represents an exact solution for the conductivity of homogeneous spheres in a homogeneous continuous medium [13]. Maxwell's model makes no assumptions as to the geometric configuration of the particles [7], but it does assume that the particles are far enough apart so that the temperature perturbations due to the particles do not interfere with the surrounding particle temperature distributions [17], this implies a low particle-volume fraction. Maxwell's model is given as

$$
\frac{k_{e}}{k_{m}}=\frac{1+2 \beta \phi}{1-\beta \phi} .
$$

The reduced form of Maxwell's equation (Eq. (1)) is included for comparison as it is the ETC model used for PARFUME modeling. Setting $k_{p}=0$ in Eq. (5) results in Eq. (1).

Effective Medium Theory (EMT) is also a commonly-used model [9]. The EMT model makes no assumption about constituent phases and assumes a random distribution of the phases [9, 12, 17]. The most common version of the EMT model is the Bruggeman model which treats the local conductivities as fluctuations about the conductivity of a uniform medium [15]. In this model there is no distinction between the continuous (matrix) and dispersed (particles) phases, and is generally considered more valid for $\square>>0$. The Bruggeman form, which has shown to be in good agreement with data for several solidsolid compounds [18], of the EMT model is given as:

$$
\frac{k_{e}}{k_{m}}=\kappa A+\sqrt{\kappa^{2} A^{2}+\kappa / 2}
$$

where $A$ is defined as:

$$
A=\frac{1}{4}\left(3 \phi-1+(2-3 \phi) \kappa^{-1}\right) .
$$

The Chiew \& Glandt model is an improved form of Maxwell's equation that takes into account higher order interactions between particles and is correct to order $\square^{2}$ [16]. This model matched experimental data for materials with $\kappa$ ranging from $10^{-3}$ to $10^{4}$ and $\square$ from 0.15 to 0.85 very well. Using the best fitting parameters by Gonzo [19], the Chiew \& Glandt model becomes:

$$
\frac{k_{e}}{k_{m}}=\frac{1+2 \beta \phi+\left(2 \beta^{3}-0.1 \beta\right) \phi^{2}+\phi^{3} 0.05 \exp (4.5 \beta)}{1-\beta \phi}
$$

\subsection{ETC finite element modeling}


Due to the complex composite nature of TRISO fuel, a complex model is required to capture the effects of all constituent components. In order to apply 2-phase analytical ETC models to TRISO fuel and to simplify the full FEA models discussed below, a single $\operatorname{ETC}_{\mathrm{p}}$ value for the particle $\left(k_{p}\right)$ is required in place of the five thermal conductivity values pertaining to each of the constituents that compose the TRISO particle. Therefore, ETC is now discussed in relation to the particle, distinguished as $\mathrm{ETC}_{\mathrm{p}}$, as well as the overall compact. Stainsby [7] applied the same method used to derive Maxwell's equation to calculate $\mathrm{ETC}_{\mathrm{p}}$ of a single particle (for the parameters shown in Table 1) as $4.13 \mathrm{~W} \cdot \mathrm{m}^{-1} \cdot \mathrm{K}^{-1}$. Essentially, the derivation involves using an initial temperature profile of a particle within an external temperature gradient. The $\mathrm{ETC}_{\mathrm{p}}$ of the particle is calculated based on the perturbation to the far-field temperature profile caused by the particle.

Table 1. Nominal values used for dimensions and thermal conductivity of each layer $[3,7,20]$

\begin{tabular}{llcc}
\hline Region & \multicolumn{1}{c}{ Material } & $\begin{array}{c}\text { Outer } \\
\text { Diameter }(\boldsymbol{\mu m})\end{array}$ & $\begin{array}{c}\text { Thermal Conductivity } \\
\left(\mathbf{W} \cdot \mathbf{m}^{-\mathbf{1}} \cdot \mathbf{K}^{-\mathbf{1}}\right)\end{array}$ \\
\hline Kernel & Uranium Dioxide & 500 & 3.7 \\
Coating 1 & Porous Pyrolytic Carbon (Buffer) & 690 & 0.5 \\
Coating 2 & Inner Pyrolytic Carbon & 770 & 4.0 \\
Coating 3 & Silicon Carbide & 840 & 16.0 \\
Coating 4 & Outer Pyrolytic Carbon & 920 & 4.0 \\
\hline
\end{tabular}

To verify using an $\mathrm{ETC}_{\mathrm{p}}$ value of $4.13 \mathrm{~W} \cdot \mathrm{m}^{-1} \cdot \mathrm{K}^{-1}$ for the TRISO particle, COMSOL Multiphysics FEA models based upon the SC, BCC, and FCC unit cells were created using a layered TRISO particle, composed of all individual components, and a homogeneous (non-layered) particle using $\mathrm{ETC}_{\mathrm{p}}$ as shown in Fig. 2a-d. Due to the symmetry for the ordered structures, unit cell models can be used to represent the whole compact (in fact 1/8 unit cell is sufficient). All the models with layered particles used the same dimensions and properties for the layers. The values used were determined from multiple sources [2-3, 7, 20-25] and can be seen in Table 1. The FEA models were made parametric to study the effects of varying the particle-volume fraction between 20-40\%. Comparing the calculated ETC for the compact using Fourier's equation for the layered FEA models and the homogenous FEA models, the results agree well with $<1 \%$ difference. Therefore, for the parameters listed in Table 1 , an $\mathrm{ETC}_{\mathrm{p}}$ value of $4.13 \mathrm{~W} \cdot \mathrm{m}^{-1} \cdot \mathrm{K}^{-1}$ can equivalently be applied to a homogeneous particle to represent the layered TRISO particle. The ETC $_{p}$ value is used in all other modeling (FEA and analytic) where the particle thermal conductivity is required.

In reality, the particle arrangement in the compact is not an ordered structure, but is more random in nature. To develop a more accurate representation of the TRISO fuel compacts, a model was developed with random particle arrangements, again using a homogeneous particle with $\mathrm{ETC}_{\mathrm{p}}$. The geometry was based on a rectangular prism (square cross section) where the height was three times the length of the sides. In all there were 125 total particles in the model. This number was then used to determine the 
necessary height of the prism for different particle-volume fractions. A computer code was written to generate the random locations of the particles, while ensuring no overlap with other particles. Using COMSOL Multiphysics with the MATLAB link, MATLAB scripts were written to automate the creation of the geometry of randomly located particles. Fig. 2e shows an example model of randomly distributed particles created by the procedure. In all cases presented, mesh convergence studies were performed to verify mesh independence.

For all FEA models, the procedure of calculating the ETC from the computational results was similar to the guarded-comparative heat flow technique (discussed in Section 3.1). For boundary conditions, a constant temperature was applied to the top of the model and a heat flux was applied at the bottom. The four lateral sides were insulated. The solution was calculated using a steady-state heat transfer module. The thermal conductivity was calculated by taking the average of the temperature through the crosssection of the model at two locations separated by a known distance. The heat flux and temperature gradient was used to calculate the ETC of the composite using Fourier's Law.

\section{Experimental}

\subsection{ETC measurement}

To measure the ETC of the TRISO fuel compacts, a system was developed based on the guardedcomparative-heat-flow technique with a calculated/validated uncertainty of less than $6 \%$ for sample thermal conductivity from $3-80 \mathrm{~W} \cdot \mathrm{m}^{-1} \cdot \mathrm{K}^{-1}$ over a measurement temperature range of $100-800^{\circ} \mathrm{C}$ [26-30].

In the guarded-comparative heat flow technique, a sample of unknown thermal conductivity is sandwiched between two reference samples (meter bars) of known thermal conductivity (Fig. 3). The steady-state temperature gradients are monitored with thermocouples placed at measured locations on the sample and meter bars. The temperature gradients are used to calculate the thermal conductivity using Fourier's Law. From the measured temperature gradients $(\Delta T / \Delta Z)$, cross-sectional area $\left(A_{m}\right)$, and thermal conductivity at the average temperature of the meter bars $\left(k_{m b}\right)$, the heat flow through each meter bar can be calculated. The heat flow through the sample $\left(q_{s}\right)$ is calculated as the average of the heat flows in the meter bars (Eq. (9)). Using the calculated heat flow in the sample from Eq. (9) and the measured temperature gradient of the sample, the ETC of the sample, at the average temperature of the sample, can be calculated from Eq. (10).

$$
\begin{aligned}
& q_{s}=\left[k_{m b}\left(\overline{T_{1}}\right) A_{m} \frac{\Delta T_{1}}{\Delta z_{1}}+k_{m b}\left(\overline{T_{2}}\right) A_{m} \frac{\Delta T_{2}}{\Delta z_{2}}\right] / 2 \\
& k_{s}\left(\overline{T_{s}}\right)=q_{s} A_{m} \frac{\Delta z_{s}}{\Delta T_{s}}
\end{aligned}
$$


Extensive effort was devoted to validate the measurement uncertainty to be $<6 \%$ and improve the guarded-comparative-heat-flow technique using measurements of certified reference materials and FEA [31-33]. Using this system, the ETC of multiple surrogate TRISO fuel compact samples and matrix-only samples have been measured and presented in Section 4.2.

\subsection{Samples}

Two different types of samples were measured. Six matrix-only samples provided by Oak Ridge National Laboratory (ORNL) and four surrogate TRISO fuel samples provided by Babcock and Wilcox. The TRISO particles in the surrogate samples contain zirconium dioxide $\left(\mathrm{ZrO}_{2}\right)$ as the kernel with standard TRISO coatings. The surrogate sample dimensions were $12.4 \mathrm{~mm}$ in diameter and $30 \mathrm{~mm}$ long. The information provided about the samples stated they were pressed at a targeted particle-volume fraction of $46 \%$ and a matrix density goal of $1.75 \mathrm{~g} / \mathrm{cm}^{3}$ from lot 13015 . The final estimated density and particle-volume fraction were $1.72 \mathrm{~g} / \mathrm{cm}^{3}$ and $47.7 \%$, respectively. The matrix consists of $80 \mathrm{wt} \%$ natural flake graphite (Asbury 3482) and 20wt\% synthetic graphite (GrafTech GP-60) bonded in Plenco 14838 resin with 7wt\% hexamethylenetetramine (HMTA) as a hardener.

The matrix-only samples arrived with a diameter and length of approximately $25 \mathrm{~mm}$ and $60 \mathrm{~mm}$, respectively. They were machined to match the dimensions of the surrogate samples. The information about the matrix-only samples can be found in Table 2 .

Table 2. Sample descriptions of measured matrix-only and TRISO-surrogate compacts

\begin{tabular}{|c|c|c|c|c|}
\hline Type & Sample ID & Matrix ID & Resin ID & $\begin{array}{c}\text { Density } \\
\left(\mathrm{g} / \mathrm{cm}^{3}\right)\end{array}$ \\
\hline \multirow{5}{*}{$\begin{array}{l}\text { Matrix Only } \\
(\text { ORNL) }\end{array}$} & RDKRS-Z016 & $R D K R S$ & Hexion Durite SC-1008 & $1.6-1.65$ \\
\hline & $R D K R S-Z 050$ & $R D K R S$ & Hexion Durite SC-1008 & $1.6-1.65$ \\
\hline & $A R B-B 1-Z 008$ & Blend I & $\begin{array}{c}\text { Hexion Durite SD-1708 w/5\% } \\
\text { HMTA }\end{array}$ & $\sim 1.75$ \\
\hline & $A R B-B 1-Z 030$ & Blend I & $\begin{array}{c}\text { Hexion Durite SD-1708 w/5\% } \\
\text { HMTA }\end{array}$ & $\sim 1.75$ \\
\hline & $\begin{array}{l}A R B-B 3-S 1 \\
A R B-B 3-S 2\end{array}$ & $\begin{array}{l}\text { Blend III } \\
\text { Blend III }\end{array}$ & $\begin{array}{l}\text { Plenco } 14838 w / 5 \% \text { HMTA } \\
\text { Plenco } 14838 w / 5 \% \text { HMTA }\end{array}$ & $\begin{array}{l}\sim 1.75 \\
\sim 1.75\end{array}$ \\
\hline $\begin{array}{c}\text { TRISO-coated } \mathrm{ZrO}_{2} \\
\text { Surrogate } \\
\varphi=47.7 \% \\
\text { (Babcock \& Wilcox) }\end{array}$ & $\begin{array}{l}13015 A \\
13015 B \\
13015 C \\
13015 D\end{array}$ & $\begin{array}{c}\text { 80wt } \% \text { Asbury } 3482 \text { and } \\
\text { 20wt \% GrafTech GP-60 }\end{array}$ & $\begin{array}{c}\mathrm{I} \\
\text { Plenco } 14838 \mathrm{w} / 7 \% \text { HMTA }\end{array}$ & $\begin{array}{c}1 \\
1.72 \\
1\end{array}$ \\
\hline
\end{tabular}

\section{Results and discussion}

\subsection{Numerical}

To understand the effect of particle constituents on ETC, a parametric study was performed to investigate the effects each layer has on $\mathrm{ETC}_{\mathrm{p}}$ of the particle using the model described in Section 2.2 from [7]. Fig. 4 shows ETC $_{\mathrm{p}}$ of the TRISO particle when the thermal conductivity of each layer is varied 
individually, assuming no resistances or gaps between layers. Nominal values of particle geometry and constituent thermal conductivity are shown in the figure. Fig. 4a uses nominal values reported from multiple sources [3, 7, 20], while Fig. $4 \mathrm{~b}$ uses values from refs. [11, 25] which has larger nominal values for most of the layers. In these results, the resulting sensitivity of $\mathrm{ETC}_{\mathrm{p}}$ to layer thermal conductivity may be understood from the gradients of each curve. For any value of thermal conductivity, the trend of the slopes is from the highest at the outer layer to the lowest at the kernel. From this perspective, the outer layer clearly has the greatest effect on the $\mathrm{ETC}_{\mathrm{p}}$ of a single particle as it has the highest sensitivity.

From the viewpoint of possible values of layer thermal conductivities, other than the outermost layer, the $\mathrm{SiC}$ layer (second from outmost) is the only layer that could realistically have thermal conductivity high enough to impact $\mathrm{ETC}_{\mathrm{p}}$. Depending on material structure, $\mathrm{SiC}$ can have thermal conductivity of $>$ $380 \mathrm{~W} \cdot \mathrm{m}^{-1} \cdot \mathrm{K}^{-1}[2]$.

Continuing this discussion, Fig. 4 also demonstrates the relative insignificance of the kernel thermal conductivity. Thus, measurement of surrogate compacts (having surrogate material in place of the fuel kernel in the TRISO particles) should have very little impact on overall compact ETC prior to being placed in reactor. To further illustrate this point, it is helpful to consider a particle in a temperature field causing a heat flux to pass across the particle. From the perspective of a thermal resistance network, one can consider conductive paths following each layer around the particle as well as a conductive path through all layers, passing through the center of the particle. From an over-simplistic standpoint, each conductive path may be viewed as being in parallel so that paths of relatively large resistance may be neglected. As stated previously, layer-layer interfacial thermal resistances and voidspace/gaps inbetween particle layers have been neglected. The effect of a gap would be equivalent to adding a layer of high resistance. As indicated by the results in Fig. 4, such a resistance would only further mitigate the effects of inner layers, thus reinforcing the importance of the outer layers in determining the overall ETC of the compacts. On the other hand, such gaps would be extremely important when calculating the temperature profile within a single particle.

As a means of distinguishing the most appropriate analytical model to represent the ETC of TRISO compacts, the FEA models discussed in Section 2.2 were used to estimate the ETC for various particlevolume fractions. The results of the FEA calculations were compared to the ETC models presented in Section 2.1. In all cases, the $\mathrm{ETC}_{\mathrm{p}}$ of a TRISO particle is taken as $4.13 \mathrm{~W} \cdot \mathrm{m}^{-1} \cdot \mathrm{K}^{-1}$ as described in Section 2.2. A comparison of these results is plotted in Fig. 5 as a function of particle-volume fraction and for various matrix thermal conductivities. The models based on a unit cell all followed the full Maxwell equation. This result is expected as the well-defined unit cell configuration maintains defined spacing between particles, which allows the particles to have less of a combinatory effect on heat transport in the material. 
The results of the randomly-distributed model, which should better represent the true TRISO particle distribution, most closely follow the Chiew \& Glandt model. For the case of matrix thermal conductivity of $30 \mathrm{~W} \cdot \mathrm{m}^{-1} \cdot \mathrm{K}^{-1}$ and a particle $\mathrm{ETC}_{\mathrm{p}}$ of $4.13 \mathrm{~W} \cdot \mathrm{m}^{-1} \cdot \mathrm{K}^{-1}$, shown in Fig. 5 , the differences between the studied analytical ETC models and the random FEA model are shown in Table 3. From Fig. 5 and this table, the Chiew \& Glandt model shows the least deviation from the random results over the entire particle-volume fraction range, with deviations $<0.6 \%$. All other analytical models deviate more from the random FEA model results with increasing deviation at higher particle-volume fractions. These differences increase as the ratio of matrix-to-particle thermal conductivity increases. Comparing the German (reduced Maxwell's) equation with the Chiew \& Glandt model for a matrix conductivity of 30 $\mathrm{W} \cdot \mathrm{m}^{-1} \cdot \mathrm{K}^{-1}$ the German equation will under predict the ETC of the fuel compact by $\sim 15 \%$ at a $40 \%$ particle-volume fraction and $\sim 20 \%$ at a $50 \%$ particle-volume fraction. Based on these results, the Chiew $\&$ Glandt model better approximates the ETC of the fuel compact than the reduced Maxwell (German) equation.

Table 3. Comparison of the ETC results for the random FEA model at various particle-volume fractions to each of the analytical ETC models. All results are for a matrix thermal conductivity of $30 \mathrm{~W} \cdot \mathrm{m}^{-1} \cdot \mathrm{K}^{-1}$ and a particle $\mathrm{ETC}_{\mathrm{p}}$ of $4.13 \mathrm{~W} \cdot \mathrm{m}^{-1} \cdot \mathrm{K}^{-1}$.

\begin{tabular}{|c|c|c|c|c|c|c|c|c|c|}
\hline \multirow{2}{*}{$\begin{array}{c}\text { Volume } \\
\text { Fraction } \\
(\square)\end{array}$} & \multirow{2}{*}{$\begin{array}{c}\text { Calculated } \\
\text { ETC } \\
\left(\mathbf{W} \cdot \mathbf{m}^{-1} \cdot \mathbf{K}^{-1}\right)\end{array}$} & \multicolumn{2}{|c|}{ Maxwell's Eqn. } & \multicolumn{2}{|c|}{$\begin{array}{c}\text { Reduced } \\
\text { Maxwell's Eqn. }\end{array}$} & \multicolumn{2}{|c|}{ Chiew \& Glandt } & \multicolumn{2}{|c|}{ EMT } \\
\hline & & ETC & $\%$ Diff. & ETC & $\%$ Diff. & ETC & $\%$ Diff. & ETC & \% Diff. \\
\hline 0.18 & 23.688 & 23.908 & -0.92 & 22.569 & 4.96 & 23.827 & -0.59 & 23.579 & 0.46 \\
\hline 0.23 & 22.116 & 22.360 & -1.09 & 20.717 & 6.75 & 22.230 & -0.51 & 21.847 & 1.23 \\
\hline 0.27 & 20.922 & 21.161 & -1.13 & 19.295 & 8.43 & 20.986 & -0.31 & 20.484 & 2.14 \\
\hline 0.30 & 19.986 & 20.285 & -1.47 & 18.261 & 9.45 & 20.072 & -0.43 & 19.476 & 2.62 \\
\hline 0.349 & 18.652 & 18.906 & -1.34 & 16.641 & 12.08 & 18.624 & 0.15 & 17.876 & 4.34 \\
\hline 0.35 & 18.636 & 18.867 & -1.22 & 16.596 & 12.29 & 18.583 & 0.29 & 17.830 & 4.52 \\
\hline 0.398 & 17.176 & 17.547 & -2.11 & 15.058 & 14.06 & 17.188 & -0.07 & 16.291 & 5.43 \\
\hline
\end{tabular}

\subsection{Experimental}

The results from the six matrix-only samples and four surrogate samples are plotted in Fig. 6 with estimated uncertainty of $<6 \%$. The results are plotted with the German matrix data [5] and a calculated ETC of the German matrix using Eq. (1) with a $47.7 \%$ particle-volume fraction for comparison. The German matrix has a higher thermal conductivity than the matrix-only samples measured in this work which is due to the differences in materials and fabrication methods described by Gontard [5]. The variations between the ARB-B1 and ARB-B3 matrix-only samples is likely due to the hardener used in the resin of these particular samples. The resin becomes too hard resulting in brittle samples. Multiple circumferential cracks were observed on these samples (see Fig. 7). Similar cracks were also observed 
during tests at ORNL so x-rays were taken and cracks can be clearly seen throughout the sample [34]. The presence of these cracks can have significant effect on bulk thermal conductivity acting as barriers to the heat flow by adding additional thermal resistance. The average effect will be to cause a reduction of the measured thermal conductivity. The surrogate samples were made using the same resin so the variation seen between the various samples could be explained by the same means.

As seen from Fig. 6, the surrogate samples have a larger thermal conductivity than the matrix-only samples, contrary to expectation. For the case of low thermal conductivity of the dispersed phase, the ETC of the TRISO compact should be less than the matrix material, as shown by the modeling results in Fig. 5. The measured matrix-only thermal conductivity is expected artificially lower due to cracking, however, it still does not explain the discrepancy between matrix-only and surrogate measured values.

To achieve such high ETC with the measured thermal conductivity of matrix-only samples would require the TRISO particle to have an $\mathrm{ETC}_{\mathrm{p}}$ of $\sim 100 \mathrm{~W} \cdot \mathrm{m}^{-1} \cdot \mathrm{K}^{-1}$. From Fig. 4 , such a magnitude would most likely be reached for an outer layer of extremely high thermal conductivity. The estimated ETC of the particles could be different than the homogenized value of $4.13 \mathrm{~W} \cdot \mathrm{m}^{-1} \cdot \mathrm{K}^{-1}$ used for the particles in this research. The largest reported measured results of TRISO layers (shown in Fig. 4b) taken from [11, 25], which are much higher than reported in $[3,7,20]$, were used to investigate the extreme case for the particle ETC. Using these values the ETC of the particle could be as high as $18.5 \mathrm{~W} \cdot \mathrm{m}^{-1} \cdot \mathrm{K}^{-1}$, which is significantly higher than $4.13 \mathrm{~W} \cdot \mathrm{m}^{-1} \cdot \mathrm{K}^{-1}$, but not high enough to explain the results in Fig. 6.

One hypothesis of the higher ETC of the surrogate samples than the matrix-only samples, which is supported by current evidence, could be related to the high anisotropy of graphite thermal conductivity [35-36]. The thermal conductivity of the graphite material may have different microstructures in the surrogate samples than in the matrix-only samples. Their individual fabrication processes provides some understanding of these differences. The matrix-only samples are made by cold compacting loose matrix powder into slugs; breaking the slugs into granules, and compacting the granules into the final compact form. Prior to compaction, the graphite flake orientation in the matrix-only compacts are random, but during compaction the flake has limited opportunity to become oriented along flow streams and as a result the flakes are largely perpendicular to the compression axis. This would result in the axial thermal conductivity being lower than the radial conductivity. In the case of the surrogate TRISO compacts, the fabrication process consists of the TRISO particles being overcoated with a graphite/resin composite matrix in a mechanically fluidized bed. The TRISO particles are spun in a bowl, while being repeatedly wetted by a water mist and rolled through a cloud of dispersed matrix powder. The graphite flake is rolled onto the TRISO particles; lying flat on the surface. The matrix overcoating of the TRISO particle then primarily has graphite flakes oriented concentrically. The graphite/resin overcoating thickness depends on the desired particle-volume fraction. The overcoated particles are then poured into a mold and 
compressed into the final compact [37-39]. This overcoating process affects the structural orientation of the graphite matrix around the TRISO particles (see Figures 13-15 in reference [40] or Fig. 8). The resulting orientation of graphite around the particles could result in preferential alignment of graphite particles providing a higher thermal conductivity "layer" around the particles. As the outer layer has the greatest impact on the thermal conductivity of the TRISO particles as discussed in Section 4.1, the fabrication procedure may result in a higher ETC for the continuous phase (graphite matrix) in the surrogate compacts than when the graphite matrix is formed solitarily.

Fig. 6 shows that the German matrix data has similar thermal conductivity values as the surrogate samples, but when the correction factor (Eq. (1)) is applied the estimated ETC of the fuel compact is less than half the measured ETC of the surrogate samples. Using the improved Chiew and Glandt model also results in a significant decrease of ETC. The experimental evidence indicates that such a dramatic decrease of ETC due to the particles is not the reality. In fact, the results of these measurements change the approach to understanding the constituent effect on ETC of TRISO compacts.

The purpose of an analytic ETC model for the TRISO compact would be to take measured values of matrix thermal conductivity, which could be done in simpler fashion using commercial property measurement systems such as laser flash, and simply calculate the overall ETC accounting for particlevolume fraction and thermal conductivity. However, due to the high anisotropy of graphite and differences inherent to the fabrication processes, it would be very difficult to create a matrix-only sample that is representative of the average matrix thermal conductivity in the composite TRISO compact.

Instead, the most appropriate method for determining the ETC of the fuel is to directly measure it for a given matrix material over a range of particle-volume fractions using a technique such as that used in this work [26, 31, 41]. As discussed above, the ETC of the matrix in a TRISO compact is a function of particle-volume fraction due to the fabrication process. Instead of using the proposed Chiew and Glandt ETC model [19] to estimate the composite ETC value of the compact, it should be used to estimate the contribution of the matrix material to the overall ETC of the TRISO fuel compact.

To illustrate this procedure, the experimentally measured ETC results for the surrogate TRISO fuel compacts in Fig. 6 can be used to estimate the effective matrix contribution to ETC of the compact. Using an average of the measured ETC of the surrogate fuel compacts $\left(k_{e}\right)$ in Fig. 6 and an $\mathrm{ETC}_{\mathrm{p}}$ for the surrogate $\mathrm{ZrO}_{2}$ particles of $4.13 \mathrm{~W} \cdot \mathrm{m}^{-1} \cdot \mathrm{K}^{-1}\left(k_{p}\right)$, the thermal conductivity contribution of the matrix $\left(k_{m}\right)$ in the surrogate TRISO fuel compacts measured in this work can be calculated using the Chiew and Glandt model (Eq. (8)). These results are shown in Fig. 9. The results show a much higher effective conductivity value for the matrix material than is measured from the matrix-only samples ( 3 times greater), which is accounted to the likely preferential aligning of the anisotropic graphite around the particles. Such an approach can be used to compare the effective contribution of different matrix graphite blends to overall 
ETC thermal conductivity of the compacts for varied particle-volume fractions as well as for variations of compact fabrication processes.

\section{Conclusions}

Numerical modeling of the ETC of the TRISO fuel compacts was performed to find a more appropriate analytical effective conductivity model that matches the geometry of the fuel compact. The numerical modeling found that the ETC of the fuel compacts is predicted most accurately by the Chiew \& Glandt model than any other models tested, with $>10 \%$ better accuracy than the model currently used (less valid for higher particle-volume fractions). The outer layer of the TRISO particle has the greatest impact on overall ETC while the kernel material has little to no impact from a practical standpoint.

The guarded-comparative heat flow technique was used to measure the effective thermal conductivity of multiple surrogate TRISO fuel compacts and matrix-only compacts. The measured results revealed that the surrogate compacts had a higher average thermal conductivity $\left(50-30 \mathrm{~W} \cdot \mathrm{m}^{-1} \cdot \mathrm{K}^{-1)}\right.$ than the matrix-only values $\left(25-15 \mathrm{~W} \cdot \mathrm{m}^{-1} \cdot \mathrm{K}^{-1}\right)$ over a temperature range of $50-600^{\circ} \mathrm{C}$. Both measurements represent axial thermal conductivity. The unexpected higher ETC values for the surrogates than the matrix-only samples is attributed to the manufacturing processes, specific to the composite form (surrogate), which affects the microscopic anisotropy of the graphite flake resulting in a higher macroscopic isotropy of thermal conductivity in the surrogates due to the interleaving of sample regions. These results underscore the importance of measuring the composite form of the compact directly. Separate measurements of the constituent matrix material will not provide representative values of the matrix in composite form. Therefore, the proposed approach to understanding ETC of the TRISO compacts is to measure it directly in composite form and then use the Chiew \& Glandt model to calculate the matrix equivalent conductivity in a TRISO fuel compact.

For future work, samples of a range of particle-volume fractions need to be measured to validate whether this new methodology can be used to measure a sample at one particle-volume fraction and extend the results to a range of particle-volume fractions. The effect of anisotropic properties in the TRISO layers will also be studied. In any case, the results of this work illustrate the importance of direct measurement of the ETC of the TRISO fuel compacts or other composites composed of constituents with anisotropic properties.

\section{Acknowledgements}

The work is supported by U.S. Department of Energy, Office of Nuclear Energy, under DOE Idaho Operations Office, contract DE-AC07-05ID14517. Work performed by Colby Jensen was supported under a Department of Energy Nuclear Energy University Programs Graduate Fellowship. 


\section{References}

[1] Grover, S. B.; Petti, D. A. In Initial Irradiation of the First Advanced Gas Reactor Fuel Development and Qualifiication Experiment in the Advanced Test Reactor, GLOBAL 2007, 2007.

[2] Snead, L. L.; Nozawa, T.; Katoh, Y.; Byun, T.-S.; Kondo, S.; Petti, D. A., J. Nucl. Mater. 2007, 371 (1), 329377.

[3] Miller, G.; Petti, D.; Maki, J.; Knudson, D. PARFUME Theory and Model Basis Report; INL/EXT-08-14497; Idaho National Laboratory: 2009.

[4] Collin, B. P., J. Nucl. Mater. 2014, 451 (1-3), 65-77.

[5] Gontard, R.; Nabielek, H. Performance Evaluation of Modern HTR TRISO Fuels; HTA-IB-05/90; 1990.

[6] Maxwell, J. C., A Treatise on Electricity and Magnetism. Clarendon press: 1881; Vol. 1.

[7] Stainsby, R.; Grief, A.; Worsley, M.; Dawson, F. Investigation of Local Heat Transfer Phenomena in a Pebble Bed HTGR Core; NR001/RP/002 R01; AMEC Nuclear: 2009.

[8] Li, D.; Sun, X.; Khaleel, M., Metall. Mater. Trans. A 2013, 44 (1), 61-69.

[9] Wang, J.; Carson, J. K.; North, M. F.; Cleland, D. J., Int. J. Heat Mass Tran. 2006, 49 (17), 3075-3083.

[10] Bari, K.; Khan, S. Z.; Lowe, T.; Farooqi, J. K., J. Mater. Sci. 2013, 48 (14), 4866-4875.

[11] Bari, K.; Osarinmwian, C.; López-Honorato, E.; Abram, T. J., Nucl. Eng. Des. 2013, 265, 668-674.

[12] Floury, J.; Carson, J.; Pham, Q. T., Food Bioprocess Technol. 2008, 1 (2), 161-170.

[13] Progelhof, R.; Throne, J.; Ruetsch, R., Polym. Eng. Sci. 1976, 16 (9), 615-625.

[14] Tsotsas, E.; Martin, H., Chem. Eng. Process: Process Intensification 1987, 22 (1), 19-37.

[15] Bruggeman, D., Ann. Phys. 1935, 24 (7), 636-664.

[16] Chiew, Y.; Glandt, E., J Colloid Interf Sci 1983, 94 (1), 90-104.

[17] Carson, J. K.; Lovatt, S. J.; Tanner, D. J.; Cleland, A. C., Int. J. Heat Mass Tran. 2005, 48 (11), 2150-2158.

[18] Raghavan, V.; Martin, H., Chem. Eng. Process: Process Intensification 1995, 34 (5), 439-446.

[19] Gonzo, E. E., Chem. Eng. J. 2002, 90 (3), 299-302.

[20] Powers, J. J.; Wirth, B. D., J. Nucl. Mater. 2010, 405 (1), 74-82.

[21] Fink, J., J. Nucl. Mater. 2000, 279 (1), 1-18.

[22] Magampa, P.; Manyala, N.; Focke, W., J. Nucl. Mater. 2013, 436 (1), 76-83.

[23] Hales, J.; Williamson, R.; Novascone, S.; Perez, D.; Spencer, B.; Pastore, G., J. Nucl. Mater. 2013, 443 (1), 531-543.

[24] DeMange, P.; Marian, J.; Caro, M.; Caro, A., J. Nucl. Mater. 2010, 405 (2), 144-155.

[25] López-Honorato, E.; Chiritescu, C.; Xiao, P.; Cahill, D. G.; Marsh, G.; Abram, T., J. Nucl. Mater. 2008, 378 (1), 35-39.

[26] Jensen, C.; Xing, C.; Folsom, C.; Ban, H.; Phillips, J., Int. J. Thermophys. 2012, 33 (2), 311-329.

[27] Ballard, S. S.; McCarthy, K. A.; Davis, W. C., Rev. Sci. Instrum. 1950, 21 (11), 905-907.

[28] Morris, R. G.; Hust, J. G., Phys. Rev. 1961, 124 (5), 1426.

[29] Pillai, C.; George, A., Int. J. Thermophys. 1991, 12 (3), 563-576.

[30] Sweet, J.; Roth, E.; Moss, M.; Haseman, G.; Anaya, J. Comparative Thermal Conductivity Measurements at Sandia National Laboratories; SAND86-0840; Sandia National Laboratory: 1986.

[31] Xing, C.; Folsom, C.; Jensen, C.; Ban, H.; Marshall, D. W., Meas Sci Technol 2014, 25 (5), 055602.

[32] Xing, C.; Jensen, C.; Folsom, C.; Ban, H.; Marshall, D. W., Appl. Therm. Eng. 2013, 62 (2), 850-857.

[33] Xing, C.; Jensen, C.; Folsom, C.; Ban, H.; Marshall, D. W., Appl. Therm. Eng. 2013, 59 (1-2), 504-514.

[34] Hunn, J. D.; Trammell, M. P.; Montgomery, F. C. Data Compliation for AGR-3/4 Matrix Ring Blank Lot ARBB1; ORNL/TM-20111/272; Oak Ridge National Laboratory: 2011.

[35] Smalc, M.; Shives, G.; Chen, G.; Guggari, S.; Norley, J.; Reynolds, R. A. In Thermal Performance of Natural Graphite Heat Spreaders, ASME 2005 Pacific Rim Technical Conference and Exhibition on Integration and Packaging of MEMS, NEMS, and Electronic Systems collocated with the ASME 2005 Heat Transfer Summer Conference, American Society of Mechanical Engineers: 2005; pp 79-89.

[36] Touloukian, Y. S.; Powell, R. W.; Ho, C. Y.; Klemens, P. G. Thermophysical Properties of Matter-The TPRC Data Series. Volume 2. Thermal Conductivity -Nonmetallic Solids; DTIC Document: 1970; pp 13-82.

[37] Phillips, J. A.; Nagley, S. G.; Shaber, E. L., Nucl. Eng. Des. 2012, 251, 261-281.

[38] Pappano, P. J.; Burchell, T. D.; Hunn, J.; Trammell, M., J. Nucl. Mater. 2008, 381 (1), 25-38.

[39] Petti, D. A.; Buongiorno, J.; Maki, J. T.; Hobbins, R. R.; Miller, G. K., Nucl. Eng. Des. 2003, 222 (2), 281297.

[40] Phillips, J.; Shaber, E. Compact Process Development at Babcock \& Wilcox; INL/EXT-11-23166; Idaho National Laboratory: 2012. 
[41] Xing, C.; Jensen, C.; Ban, H.; Phillips, J., Meas. Sci. Technol. 2011, 22 (7), 075702. 
Fig. 1: Picture of TRISO fuel compact and schematic of a TRISO particle. 
Fig. 2. FEA models used for calculating ETC: a. simple cubic, b. body-centered cubic, c. face-centered cubic, d. body-centered cubic using a homogeneous particle, e. random model using homogeneous particles 
Fig. 3. Schematic of the guarded-comparative heat flow technique used to measure surrogate TRISO fuel and matrix-only compacts. 
Fig. 4. Effect of layer thermal conductivity on the ETC of a single TRISO particle. a. Nominal thermal conductivity values and layer thicknesses (denoted by the outside diameter (O.D.)) used for the individual layer properties found in $[3,7,20]$. b. Nominal values for the layer properties found in $[11,25]$. 
Fig. 5. FEA results for the ETC of the fuel compact for multiple matrix thermal conductivities $\left(\mathrm{k}_{\mathrm{m}}\right)$ compared to analytical ETC models as a function of particle-volume fraction. Particle thermal conductivity is taken as $4.13 \mathrm{~W} \cdot \mathrm{m}^{-1} \cdot \mathrm{K}^{-1}$. 
Fig. 6. Measured thermal conductivity results of matrix-only and surrogate samples plotted with legacy German data. Sample information can be found in Section 3.2. 
Fig. 7. Photograph of ARB-B3 sample illustrating appearance of cracks, primarily perpendicular to the sample axis, attributed to the resin material used in these particular samples. 
Fig. 8. Optical micrograph showing the appearance of graphite anisotropy in a fuel compact. Image used from ref. [40]. 
Fig. 9. Comparison of thermal conductivity values for matrix-only and surrogate compacts with the estimated matrix thermal conductivity (ETC) of the surrogate compact calculated using the Chiew and Glandt model with particle thermal conductivity taken as $4.13 \mathrm{~W} \cdot \mathrm{m}^{-1} \cdot \mathrm{K}^{-1}$. 


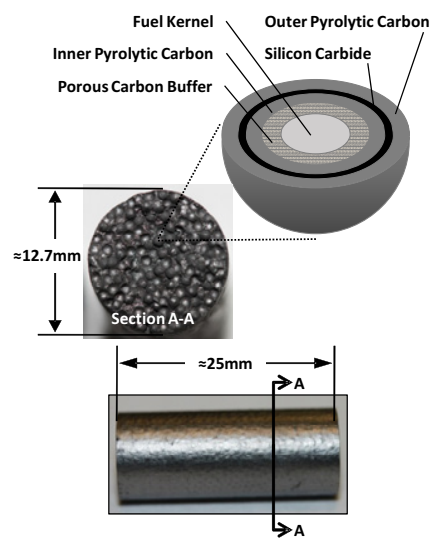




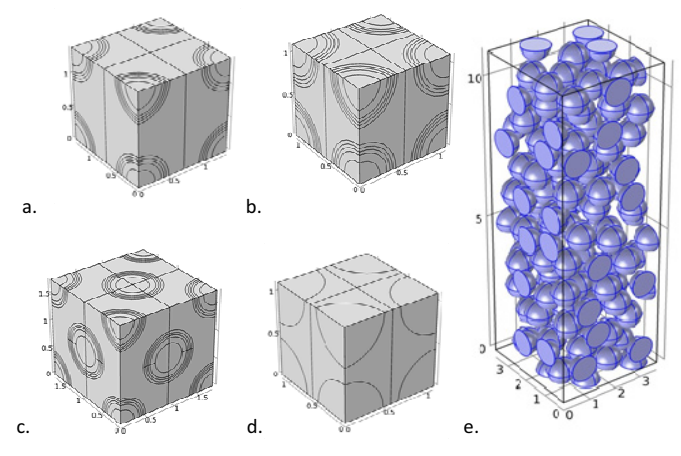




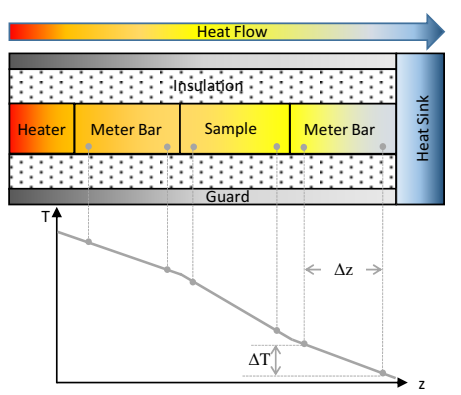




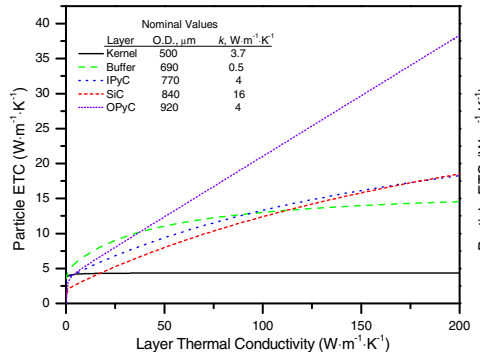

a.

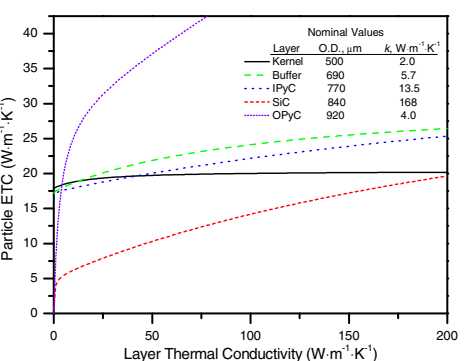

b.
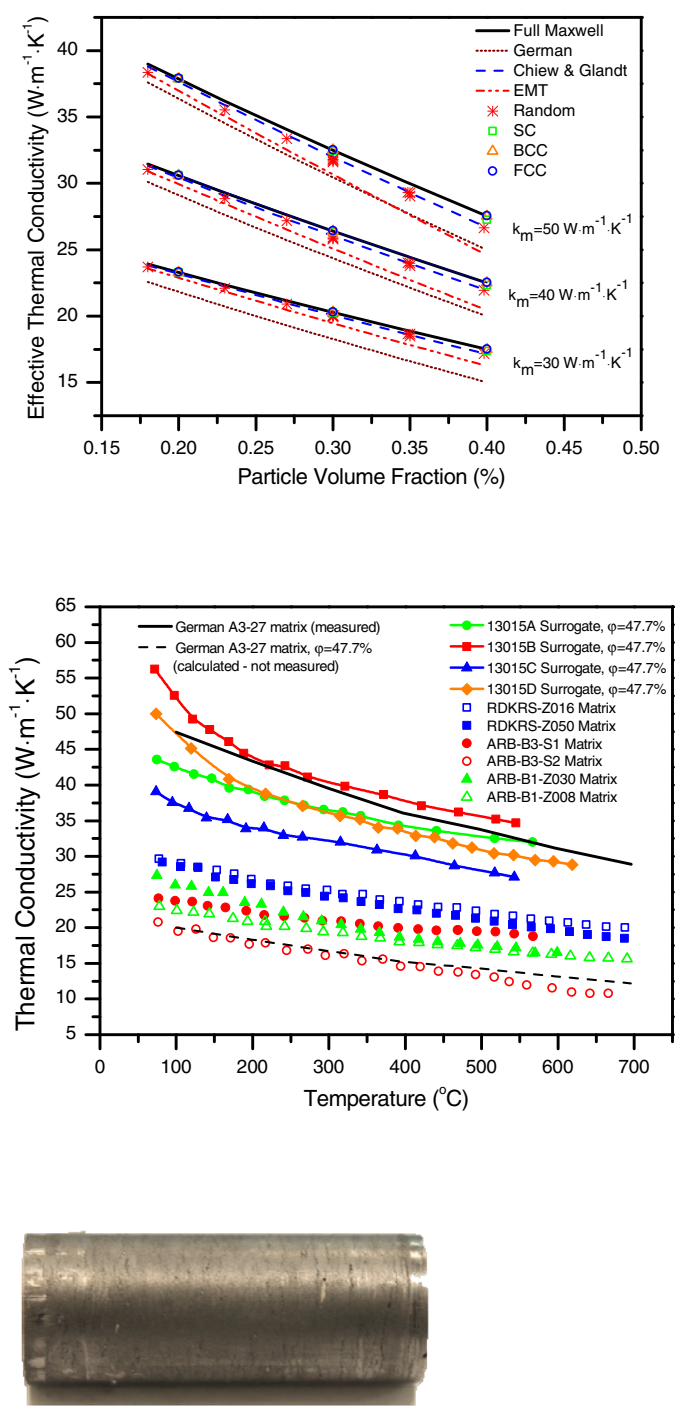

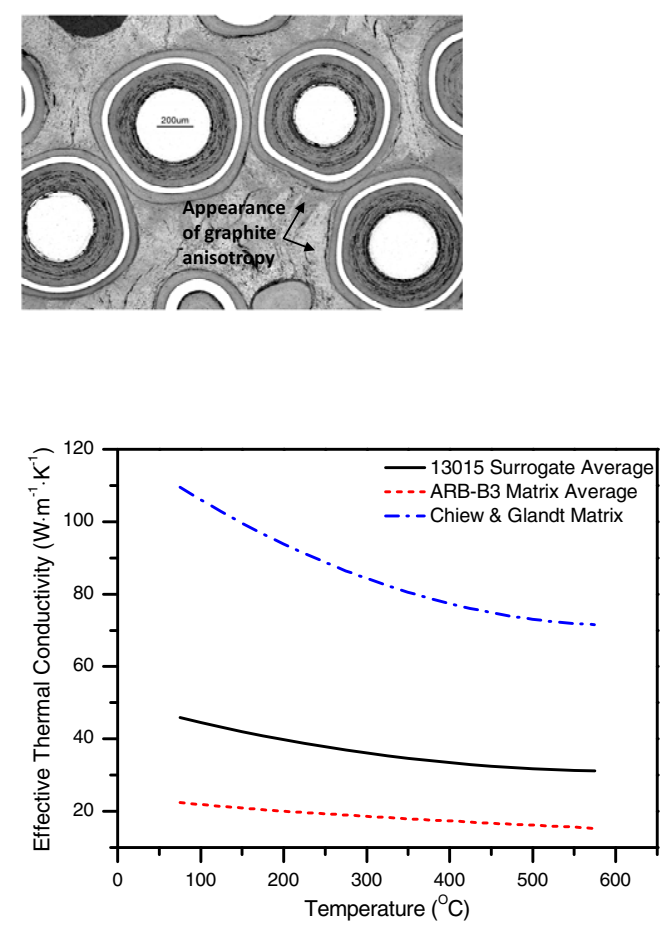